\title{
Effect of Tidal Volume and Nebulizer Type and Position on Albuterol Delivery in a Pediatric Model of Mechanical Ventilation
}

\author{
Ariel Berlinski MD and J Randy Willis RRT-NPS
}

\begin{abstract}
BACKGROUND: Optimization of factors affecting aerosol delivery during mechanical ventilation in the pediatric population is important. We hypothesized that increasing the tidal volume $\left(V_{T}\right)$, using a vibrating mesh nebulizer, and placing the nebulizer at the ventilator would increase lung dose/delivery efficiency. METHODS: Continuous-output jet and vibrating mesh nebulizers loaded with albuterol $(2.5 \mathrm{mg} / 3 \mathrm{~mL})$ were compared when placed before the $\mathrm{Y}$-piece and at the ventilator. The model consisted of a ventilator operated in pressure-regulated volume control ventilation mode at a breathing frequency of 20 breaths/min, PEEP of $5 \mathrm{~cm} \mathrm{H}_{2} \mathrm{O}, \mathrm{F}_{\mathrm{IO} 2}$ of 0.4 , inspiratory time of $0.75 \mathrm{~s}$, and bias flow of $0.5 \mathrm{~L} / \mathrm{min}$ with a humidifier $\left(37 \pm 1.5^{\circ} \mathrm{C}\right)$ and an adult heated-wired circuit. $\mathrm{V}_{\mathrm{T}}$ values of $100,150,200$, and $300 \mathrm{~mL}$ were studied. The circuit was connected in series to a 5.5-mm inner diameter endotracheal tube with a filter (lung dose) interposed between them. Delivery efficiency was calculated as a percentage of the nominal dose captured on the filter. Albuterol content was analyzed by spectrophotometry $(276 \mathrm{~nm})$. RESULTS: No differences in lung dose/delivery efficiency were found at different $V_{T}$ values for the jet nebulizer (both positions) and the vibrating mesh nebulizer (ventilator). Lung dose/delivery efficiency was higher $(P<.02)$ at a $V_{T}$ of $100 \mathrm{~mL}$ compared with the other volumes tested. The vibrating mesh nebulizer had higher lung dose/delivery efficiency compared with the jet nebulizer only when placed before the Y-piece. Moving the nebulizers from before the $Y$-piece to the ventilator increased lung dose/delivery efficiency for all conditions tested except the vibrating mesh nebulizer at a $V_{T}$ of $100 \mathrm{~mL}(P=.36)$. CONCLUSIONS: Optimization of inhaled drug delivery during pediatric mechanical ventilation should include careful selection of the type of delivery device and its placement in the ventilator circuit. Increasing $\mathbf{V}_{\mathbf{T}}$ during nebulization did not increase lung dose/delivery efficiency. Key words: tidal volume; mechanical ventilator; drug delivery; aerosol; jet nebulizer; vibrating mesh nebulizer. [Respir Care 2015;60(10):1424-1430. @ 2015 Daedalus Enterprises]
\end{abstract}

\section{Introduction}

Children receiving invasive mechanical ventilation are frequently prescribed in-line nebulized therapy. ${ }^{1,2}$ Drug

The authors are affiliated with Arkansas Children's Hospital, Little Rock, Arkansas. Dr Berlinski is also affiliated with the Pulmonology Section, Department of Pediatrics, University of Arkansas for Medical Sciences, and the Pediatric Aerosol Research Laboratory, Arkansas Children's Hospital Research Institute, Little Rock, Arkansas.

Mr Willis presented a version of this paper at the AARC Congress 2014, held December 9-12, 2014, in Las Vegas, Nevada.

Dr Berlinski has disclosed relationships with Vertex Pharmaceuticals, AbbVie, Aptalis, Genentech, Janssen Research \& Development, Gilead Sciences, Teva Pharmaceutical Industries, Philips Respironics, and Therapeutics Development Network. Mr Willis has disclosed no conflicts of delivery efficiency during mechanical ventilation is affected by several factors. ${ }^{3}$ Some of them are easy to modify (type of delivery device and position in the circuit), but others are not (endotracheal tube [ETT] size).

Although several types of devices are available, continuous-output jet and vibrating mesh nebulizers are the most

\footnotetext{
interest. The Pediatric Aerosol Research Laboratory at Arkansas Children's Hospital Research Institute was partially established by and receives partial support from the George Endowment for Asthma.

Correspondence: Ariel Berlinski MD, Pulmonology Section, Department of Pediatrics, University of Arkansas for Medical Sciences, 1 Children's Way, Slot 512-17, Little Rock, AR 72202. E-mail: berlinskiariel@ uams.edu.
}

DOI: $10.4187 /$ respcare. 04013 


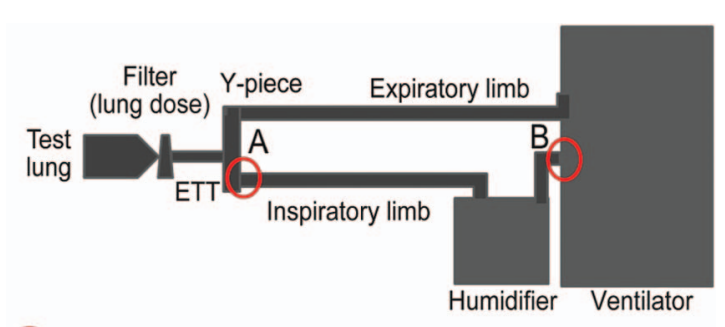

Fig. 1. Experimental setup. Position $A$ is at the inspiratory limb, before the $\mathrm{Y}$-piece, and position $\mathrm{B}$ is at the ventilator. ETT = endotracheal tube.

commonly used. The devices can be placed at different positions in the ventilator circuit, with the most commonly used being the inspiratory limb before the Y-piece and at the ventilator. ${ }^{4-9}$

Drug delivery efficiency data on adult models of mechanical ventilation suggested that higher tidal volume $\left(\mathrm{V}_{\mathrm{T}}\right)$ resulted in higher lung delivery.2,10,11 These results are in agreement with data generated using models of spontaneously breathing children and adults. ${ }^{12,13}$ This information led to the practice of increasing $\mathrm{V}_{\mathrm{T}}$ or providing sigh breaths during nebulization. ${ }^{2}$ Previous research using a pediatric model of mechanical ventilation with a jet nebulizer, ${ }^{6}$ and a clinical study in adults, ${ }^{14}$ had opposite findings. We hypothesized that increasing the $\mathrm{V}_{\mathrm{T}}$, using a vibrating mesh nebulizer, and placing the nebulizer at the ventilator would increase lung dose and drug delivery efficiency in a pediatric model of mechanical ventilation.

\section{Methods}

\section{Mechanical Ventilation Model}

This model was similar to one previously published, except we used a lower bias flow (Fig. 1).6,7 The model consisted of a ventilator (Servo-i, Maquet, Solna, Sweden) operated in pressure-regulated volume control ventilation mode at a breathing frequency of 20 breaths/min, PEEP of $5 \mathrm{~cm} \mathrm{H}_{2} \mathrm{O}, \mathrm{F}_{\mathrm{IO}_{2}}$ of 0.4 , inspiratory time of $0.75 \mathrm{~s}$, and bias flow of $0.5 \mathrm{~L} / \mathrm{min}$ with a humidifier $\left(37 \pm 1.5^{\circ} \mathrm{C}\right)$. The following $\mathrm{V}_{\mathrm{T}}$ values were studied: $100,150,200$, and $300 \mathrm{~mL}$. An adult heated-wired circuit (1.83 m long, 22-mm inner diameter [ID]; Evaqua, Fisher \& Paykel Healthcare, Auckland, New Zealand) was used. The circuit was connected in series to a 5.5-mm ID cuffed ETT (Mallinckrodt Lo-Pro, Covidien, Pleasanton, California) and a test lung (SmartLung, $600 \mathrm{~mL}$, imtmedical, Buchs, Switzerland) with a low-volume filter holder interposed between them. A new respiratory filter (PARI Respiratory Equipment, Midlothian, Virginia) was used for each run. The cuff was inflated to provide a closed circuit.

\section{QUICK LOOK}

\section{Current knowledge}

The efficiency of aerosol delivery in pediatric patients is impacted by the type of nebulizer and position in the circuit, ventilator settings, bias flow, breathing pattern, and size of the artificial airway. The optimum position for different types of nebulizers may be different.

\section{What this paper contributes to our knowledge}

In a lung model, optimization of inhaled drug delivery during simulated pediatric mechanical ventilation should include careful selection of the type of delivery device and placement in the ventilator circuit. Placement of the nebulizer before the humidifier increased lung dose and delivery efficiency in a pediatric model with low bias flow. Increasing tidal volume did not result in an increase in lung dose or delivery efficiency. Vibrating mesh nebulizers had higher lung dose and delivery efficiency compared with continuous-output jet nebulizers when placed before the Y-piece, but not when placed at the ventilator.

\section{Devices}

Four new units of a continuous-output jet nebulizer (UpDraft II Opti-Neb, Hudson RCI/Teleflex Medical, Research Triangle Park, North Carolina) and a vibrating mesh nebulizer (Aeroneb Solo, Aerogen, Galway, Ireland) were tested (Fig. 2). The jet nebulizer was connected to the circuit with a spring-loaded T-piece (AirLife Valved Tee adapter, Thayer Medical, Tucson, Arizona), and the vibrating mesh nebulizer was connected with its proprietary T-piece adapter. Ventilator settings were adjusted during jet nebulization to compensate for the increase in flow, but no changes were required during vibrating mesh nebulization. ${ }^{15}$ The devices were placed in the inspiratory limb before the Y-piece (Fig. 1, position A) and at the ventilator (position B).

\section{Procedure}

Devices were loaded with albuterol nebulizer solution (2.5 mg/3 mL; Nephron Pharmaceuticals, Orlando, Florida). At the beginning of each run, a new respiratory filter was used, the filter holder was sealed with Teflon tape, and the returned $\mathrm{V}_{\mathrm{T}}$ was checked. Jet $(6 \mathrm{~L} / \mathrm{min})$ and vibrating mesh nebulizers were operated for 5 and $15 \mathrm{~min}$, respectively. ${ }^{7}$ The length of operation was decided after preliminary testing showed that these intervals allowed complete nebulization of medication within the reservoir 


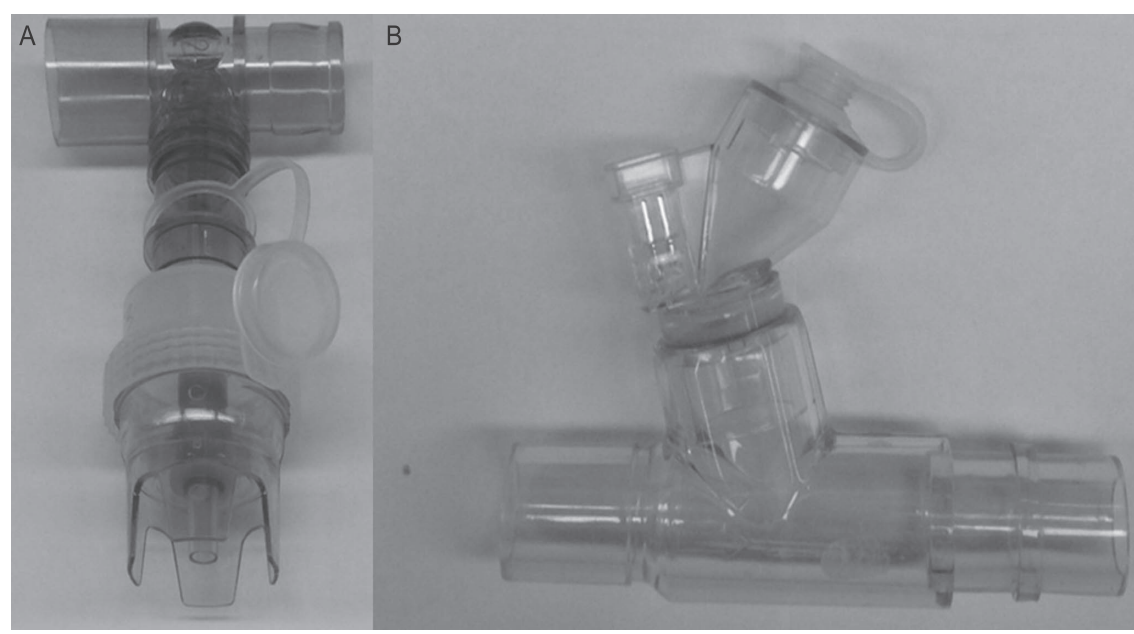

Fig. 2. Devices tested. A: Continuous-output jet nebulizer. B: Vibrating mesh nebulizer.

Table 1. Lung Dose With Different Nebulizers, Positions in the Ventilator Circuit, and $\mathrm{V}_{\mathrm{T}}$

\begin{tabular}{|c|c|c|c|c|}
\hline \multirow{3}{*}{$\mathrm{V}_{\mathrm{T}}$} & \multicolumn{4}{|c|}{ Lung Dose ( $\mu \mathrm{g}$ of albuterol) } \\
\hline & \multicolumn{2}{|c|}{ Placed Before the Y-piece } & \multicolumn{2}{|c|}{ Placed at the Ventilator } \\
\hline & $\begin{array}{l}\text { Continuous-Output } \\
\text { Jet Nebulizer }\end{array}$ & $\begin{array}{l}\text { Vibrating Mesh } \\
\text { Nebulizer }\end{array}$ & $\begin{array}{l}\text { Continuous-Output } \\
\text { Jet Nebulizer }\end{array}$ & $\begin{array}{l}\text { Vibrating Mesh } \\
\text { Nebulizer }\end{array}$ \\
\hline $100 \mathrm{~mL}$ & $102 \pm 7$ & $184 \pm 29 * \dagger$ & $218 \pm 41 \ddagger$ & $238 \pm 128 \S$ \\
\hline $150 \mathrm{~mL}$ & $93 \pm 3$ & $121 \pm 27 \dagger$ & $217 \pm 28 \ddagger$ & $367 \pm 130 \neq$ \\
\hline $200 \mathrm{~mL}$ & $90 \pm 17$ & $123 \pm 17 \dagger$ & $230 \pm 38 \ddagger$ & $326 \pm 96 \neq$ \\
\hline $300 \mathrm{~mL}$ & $79 \pm 12$ & $118 \pm 20 \dagger$ & $248 \pm 68 \ddagger$ & $321 \pm 78 \ddagger$ \\
\hline \multicolumn{5}{|c|}{$\begin{array}{l}* P<.02 \text { compared with other tidal volumes }\left(\mathrm{V}_{\mathrm{T}}\right) \text { for the same device/position. } \\
\dagger P<.04 \text { compared with the continuous-output jet nebulizer placed before the } \mathrm{Y} \text {-piece. } \\
\ddagger P<.02 \text { (continuous-output jet nebulizer) and } P<.03 \text { (vibrating mesh nebulizer) compared with the same device placed before the } \mathrm{Y} \text {-piece. } \\
\& P=0.36 \text { compared with the same device placed before the } \mathrm{Y} \text {-piece. }\end{array}$} \\
\hline
\end{tabular}

for all devices, and they were also comparable to the intervals used in our previous studies. ${ }^{6,7}$ In addition, the Aerogen control units have settings for 15 and $30 \mathrm{~min}$, and previously published data showed that extending nebulization beyond $5 \mathrm{~min}$ did not increase drug output for jet nebulizers. ${ }^{16}$

Filters were eluted with ultrapure water, and the washings were tested for albuterol concentration by spectrophotometry (BioMate 3 ultraviolet-visible spectrophotometer, Thermo Fisher Scientific, Waltham, Massachusetts) at $276 \mathrm{~nm} .{ }^{6}$ Four units of each type of aerosol generator were tested in each of the 2 positions. The amount of drug captured in the filter $(\mu \mathrm{g})$ was considered the lung dose. The delivery efficiency was calculated as: \% efficiency $=($ lung dose in $\mu \mathrm{g} / 2,500) \times 100$.

\section{Statistical Analysis}

Analysis of variance was used to compare lung doses at different $\mathrm{V}_{\mathrm{T}}$ values. The Tukey test was used for multiple- comparison testing. A $t$ test was used to compare different devices at the same position (unpaired with unequal variance) and different positions for the same device (paired). $P<.05$ was considered statistically significant. A statistical software package (KaleidaGraph 4.1, Synergy Software, Reading, Pennsylvania) was used for all calculations.

\section{Results}

Data are presented in Table 1 as lung dose ( $\mu \mathrm{g}$ of albuterol) and in Figure 3 as delivery efficiency.

\section{Effect of $V_{T}$ on Lung Dose/Delivery Efficiency}

No differences in lung dose/delivery efficiency were found at different $\mathrm{V}_{\mathrm{T}}$ values for the continuous-output jet nebulizer when it was placed either before the Y-piece $(P=.08)$ or at the ventilator $(P=.75)$. The delivery efficiency ranged between 3.6 and $4.1 \%$ and between 8.7 


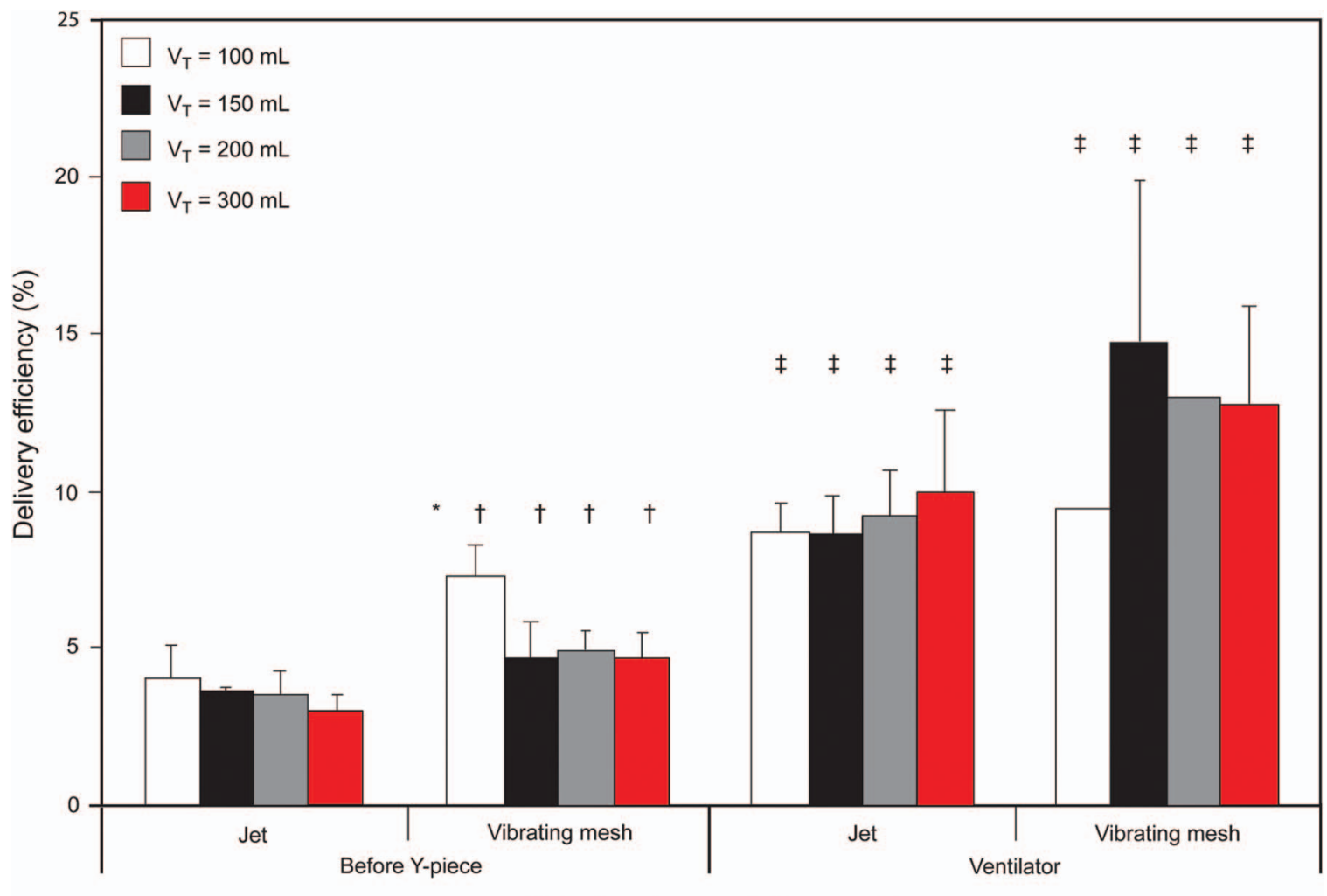

Nebulizer type and placement

Fig. 3. Delivery efficiency (percent) with different nebulizers, positions in the ventilator circuit, and tidal volumes $\left(V_{\mathrm{T}}\right)$. ${ }^{*} P<.02$ compared with other $\mathrm{V}_{\mathrm{T}}$ values for same device/position. $\dagger P<.04$ compared with the continuous-output jet nebulizer placed before the $\mathrm{Y}$-piece. $\ddagger P<.02$ (continuous-output jet nebulizer) and $P<.03$ (vibrating mesh nebulizer) compared with same device placed before the $Y$-piece.

and $9.9 \%$ for the jet nebulizer placed before the Y-piece and at the ventilator, respectively.

No differences in lung dose/delivery efficiency were found at different $\mathrm{V}_{\mathrm{T}}$ values for the vibrating mesh nebulizer when it was placed at the ventilator $(P=.44)$. However, lung dose/delivery efficiency was higher at a $\mathrm{V}_{\mathrm{T}}$ of $100 \mathrm{~mL}$ compared with the other values $(P<.02)$ when the vibrating mesh nebulizer was placed before the $\mathrm{Y}$ piece. No differences were found at the other $\mathrm{V}_{\mathrm{T}}$ values $(P<.98)$. The delivery efficiency ranged between 4.7 and $7.3 \%$ and between 9.5 and $14.7 \%$ for the vibrating nebulizer placed before the Y-piece and at the ventilator, respectively.

\section{Effect of Nebulizer Type on Lung Dose/Delivery Efficiency}

The vibrating mesh nebulizer had 1.3-1.8-fold higher lung dose/delivery efficiency compared with the continuous-output jet nebulizer when placed at the Y-piece $(P<.04)$. Although a similar trend was noted when the nebulizers were placed at the ventilator, the difference did not reach statistical significance $(P<.79)$.

\section{Effect of Nebulizer Placement on Lung Dose/Delivery Efficiency}

Moving the continuous-output jet nebulizer from the Y-piece to the ventilator increased lung dose/delivery efficiency by 2-3-fold at all $\mathrm{V}_{\mathrm{T}}$ values $(P>.02$ for each $\mathrm{V}_{\mathrm{T}}$ ). Moving the vibrating mesh nebulizer from the $\mathrm{Y}$ piece to the ventilator increased lung dose/delivery efficiency by 3 -fold at a $\mathrm{V}_{\mathrm{T}}$ of $150-300 \mathrm{~mL}(P<.03$ for each $\left.\mathrm{V}_{\mathrm{T}}\right)$. Although a similar trend was seen for a $\mathrm{V}_{\mathrm{T}}$ of $100 \mathrm{~mL}$, the difference did not reach statistical significance $(P=.36)$

\section{Discussion}

We found that moving the nebulizer from before the Y-piece to the ventilator increased lung dose/delivery efficiency in a pediatric ventilator model with low bias flow. We also found that increasing the $\mathrm{V}_{\mathrm{T}}$ did not result in an increase in lung dose/delivery efficiency. In addition, we found that vibrating mesh nebulizers had higher lung dose/delivery efficiency compared with continuous-output jet nebulizers when placed before the Y-piece but not when placed at the ventilator. 


\section{Aerosol Delivery During Pediatric Mechanical Ventilation}

$\mathbf{V}_{\mathbf{T}}$

Our results are in partial agreement with previously published data using a similar setup except that the bias flow was $2 \mathrm{~L} / \mathrm{min} .{ }^{6}$ In that study, another brand of continuousoutput jet nebulizer was studied when placed before the Y-piece and the humidifier at $\mathrm{V}_{\mathrm{T}}$ values of 100 and $200 \mathrm{~mL}$. No differences in lung dose were found between $\mathrm{V}_{\mathrm{T}}$ values at either position. Two other studies compared pediatric and adult models but used different size circuits, making the comparison more difficult. ${ }^{5,17}$ Wan et al ${ }^{17}$ compared the lung dose generated by a continuous-output jet nebulizer placed at the ventilator during different nebulization modes. The lung dose was $6.3 \%$ for the pediatric model $\left(\mathrm{V}_{\mathrm{T}}\right.$ of $160 \mathrm{~mL}$, breathing frequency of 25 breaths/min, inspiratory-expiratory ratio of $1: 2$ with a $15-\mathrm{mm}$ circuit and a 5.0-mm ID ETT) and 7.4\% for the adult model $\left(\mathrm{V}_{\mathrm{T}}\right.$ of $600 \mathrm{~mL}$, breathing frequency of 16 breaths/min, inspiratory-expiratory ratio of 1:2.5 with a $22-\mathrm{mm}$ circuit and a 7.5-mm ID ETT). Their results were similar to ours: 8.7 and $9.9 \%$ at a $\mathrm{V}_{\mathrm{T}}$ of 150 and $300 \mathrm{~mL}$, respectively. Ari et $\mathrm{al}^{5}$ compared a continuous-output jet nebulizer and a vibrating mesh nebulizer placed at the ventilator and $15 \mathrm{~cm}$ before the Y-piece on the inspiratory limb. They used an adult model $\left(\mathrm{V}_{\mathrm{T}}\right.$ of $500 \mathrm{~mL}$, breathing frequency of 20 breaths/min with a $22-\mathrm{mm}$ humidified circuit and an 8-mm ID ETT) and a pediatric model $\left(\mathrm{V}_{\mathrm{T}}\right.$ of $100 \mathrm{~mL}$, breathing frequency of 20 breaths/min with a $15-\mathrm{mm}$ humidified circuit and a 5.0-mm ID ETT). The jet nebulizer was operated at $2.5 \mathrm{~L} / \mathrm{min}$. Comparison between both models provided different results depending on the device and its placement in the ventilator circuit. No differences between the adult and pediatric models were found when the jet nebulizer was placed before the humidifier and the vibrating mesh nebulizer was placed before the Y-piece. The adult model provided higher lung dose compared with the pediatric model when the jet nebulizer was placed before the Y-piece and the vibrating mesh nebulizer was placed before the humidifier. Comparison is difficult due to the fact that both models differ not only in $\mathrm{V}_{\mathrm{T}}$ but also in the IDs of the ETT and ventilator circuit.

Our findings contradict previously published data obtained using adult models. ${ }^{10,11} \mathrm{O}$ 'Riordan et $\mathrm{al}^{10}$ found that increasing the $\mathrm{V}_{\mathrm{T}}$ from 700 to $1,000 \mathrm{~mL}$ resulted in a $12-25 \%$ increase in lung dose. Fink et $\mathrm{al}^{11}$ found a progressive increase in lung dose when the $\mathrm{V}_{\mathrm{T}}$ was increased from 100 to $800 \mathrm{~mL}$. The different results could be attributed to several differences in the investigational setups. O'Riordan et $\mathrm{al}^{10}$ evaluated different continuous-output jet nebulizers in an adult model of mechanical ventilation (ETT ID of $9 \mathrm{~mm}$ ). Fink et al ${ }^{11}$ used a metered-dose inhaler, a dry circuit, and an ETT with an ID of $8 \mathrm{~mm}$ in CPAP mode. The pressure was set at 0 , and different $V_{T}$ values were generated by the breathing simulator.
The effect of different $V_{T}$ values on lung dose/delivery efficiency depended on the placement of the vibrating mesh nebulizer in the ventilator circuit. Although no changes were noted with the device placed at the ventilator, higher lung dose/delivery efficiency was noted for the lower $\mathrm{V}_{\mathrm{T}}$ when the nebulizer was placed before the Y-piece. This could be explained by the presence of a reservoir effect due to the fact that at lower $\mathrm{V}_{\mathrm{T}}$, a higher proportion of the inhaled volume contains aerosol. A different response was reported by Ari et al, 5 who found that a vibrating mesh nebulizer placed before the Y-piece provided similar lung dose in pediatric and adult models. Conversely, they reported a higher lung dose/delivery efficiency for the adult model when the same nebulizer was placed at the ventilator. The different findings could be explained in part by the differences in the experimental setup, particularly that the nebulizer was placed immediately before the Y-piece in our study, and it was placed $15 \mathrm{~cm}$ before the Y-piece in theirs. We speculate that this difference could have decreased the importance of the reservoir effect. The clinical implications of our findings are that clinicians should not consider increasing $\mathrm{V}_{\mathrm{T}}$ as a tool to optimize aerosol delivery during pediatric mechanical ventilation because it does not provide any benefits and yet increases the risk of barotrauma and volutrauma.

\section{Delivery Device and Position}

The increase in lung dose/delivery efficiency found upon moving the continuous-output jet and vibrating mesh nebulizers from the Y-piece to the ventilator is consistent with previous reports. ${ }^{5,7} \mathrm{~A}$ similar effect was reported in a previous study for jet and vibrating mesh nebulizers (2.6- and 3.3 -fold increases, respectively). ${ }^{7}$ The experimental setup was similar except for a bias flow of $2 \mathrm{~L} / \mathrm{min}$ and a $\mathrm{V}_{\mathrm{T}}$ of $200 \mathrm{~mL} .7$ A smaller effect was reported in another study for jet and vibrating mesh nebulizers (1.2- and 1.2-fold differences, respectively). ${ }^{5}$ The experimental setup included a smaller size ventilator circuit (15-mm ID), a slightly narrower ETT (5.0-mm ID), and a higher bias flow ( $2 \mathrm{~L} / \mathrm{min})$. Our lung dose/delivery efficiency at a $\mathrm{V}_{\mathrm{T}}$ of $100 \mathrm{~mL}$ also increased by similar amounts, but the difference was not statistically significant due to high inter-device variation.

The vibrating mesh nebulizer had a larger inter-unit variation than the jet nebulizer. This is consistent with previous studies that reported a coefficient of variation $(\mathrm{SD} /$ mean $\times 100)$ for the vibrating mesh nebulizer placed at the ventilator of $25-30 \% .5,7$ Practitioners need to be aware of this phenomenon already reported for jet nebulizers. ${ }^{18}$ The findings for the continuous-output jet and vibrating mesh nebulizers were similar to those reported with adult models. ${ }^{4,5,9}$ 


\section{Aerosol Delivery During Pediatric Mechanical Ventilation}

The efficiency of the devices cannot be compared without considering their placement in the ventilator circuit. The vibrating mesh nebulizer had higher lung dose/delivery efficiency than the continuous-output jet nebulizer when placed at the Y-piece, as reported previously..$^{5,7,8}$ However, it was lower in this study (1.8-fold) than reported by Ari et $a 1,{ }^{5}$ Sidler-Moix et $a l,{ }^{8}$ and Berlinski and Willis ${ }^{7}$ (2.7-, 3.6-, and 4.4-fold, respectively). The differences in magnitude could be explained in part by differences in experimental setup. Although Sidler-Moix et $\mathrm{al}^{8}$ used a similar diameter ventilator circuit and a smaller size ETT (4.0 $\mathrm{mm}$ ID), they did not use humidification. The latter would lead to an overestimation of the delivery efficiency. ${ }^{4,8}$ Although Ari et $\mathrm{al}^{5}$ used a smaller size heated and humidified ventilator circuit (15-mm ID) and a slightly smaller size ETT (5.0 mm ID), they also used a higher bias flow ( $2 \mathrm{~L} / \mathrm{min}$ ). In a previous study, ${ }^{7}$ we used a similar experimental setup except for a higher bias flow $(2 \mathrm{~L} / \mathrm{min})$. Comparison of the data from these 2 studies with those from this study highlights the deleterious effect of bias flow on aerosol drug delivery during mechanical ventilation. ${ }^{4}$ Also, difficulties arise when extrapolating results from one setup to another.

The difference in lung dose/delivery efficiency generated by both devices did not reach statistical significance when the devices were placed at the ventilator. This is in agreement with the results obtained by Ari et al, ${ }^{5}$ who did not find differences in their pediatric model when comparing bias flows of 2 and $5 \mathrm{~L} / \mathrm{min}$. In a previous study, ${ }^{7}$ we found a significant difference using a similar setup except for a higher bias flow $(2 \mathrm{~L} / \mathrm{min})$. We speculate that the relationship between bias flow is not simple and that the size and length of the ventilator circuit, the $\mathrm{V}_{\mathrm{T}}$, and the position of the aerosol generator play a role in the final outcome (lung dose). We speculate that when using an aerosol generator at the ventilator with a $22-\mathrm{mm}$ ventilator circuit, a minimal amount of bias flow is necessary to fill up the inspiratory limb with aerosol during expiration. However, an excess of bias flow results in an increase in impaction against the walls, resulting in lower lung dose.

This study has limitations that are related to the nature of the experimental setup. The in vitro lung dose overestimates the actual dose because the filters do not allow exhalation of the aerosols that are not deposited. However, even with this limitation, this is a well-accepted methodology with already established in vivo/in vitro correlations. ${ }^{19,20}$ Also, we did not measure the particle size of the aerosols that left the tip of the ETT. However, although particle size is a major limitation in bypassing the upper airway, it is not a problem in this context because once the aerosol leaves the ETT, it has already bypassed the upper airway.

\section{Conclusions}

Optimization of inhaled drug delivery during pediatric mechanical ventilation should include careful selection of the type of delivery device and its placement in the ventilator circuit. Increasing $\mathrm{V}_{\mathrm{T}}$ during nebulization did not increase lung dose/delivery efficiency.

\section{REFERENCES}

1. Fink JB. Aerosol delivery to ventilated infant and pediatric patients. Respir Care 2004;49(6):653-665.

2. Ehrmann S, Roche-Campo F, Sferrazza Papa GF, Isabey D, Brochard L, Apiou-Sbirlea G, REVA research network. Aerosol therapy during mechanical ventilation: an international survey. Intensive Care Med 2013;39(6):1048-1056.

3. Dhand R. Aerosol delivery during mechanical ventilation: from basic techniques to new devices. J Aerosol Med Pulm Drug Deliv 2008; 21(1):45-60.

4. Ari A, Areabi H, Fink JB. Evaluation of aerosol generator devices at 3 locations in humidified and non-humidified circuits during adult mechanical ventilation. Respir Care 2010;55(7):837-844.

5. Ari A, Atalay OT, Harwood R, Sheard MM, Aljamhan EA, Fink JB. Influence of nebulizer type, position, and bias flow on aerosol drug delivery in simulated pediatric and adult lung models during mechanical ventilation. Respir Care 2010;55(7):845-851.

6. Berlinski A, Willis JR. Albuterol delivery by intrapulmonary percussive ventilator and jet nebulizer in a pediatric ventilator model. Respir Care 2010;55(12):1699-1704.

7. Berlinski A, Willis JR. Albuterol delivery by 4 different nebulizers placed in 4 different positions in a pediatric ventilator in vitro model. Respir Care 2013;58(7):1124-1133.

8. Sidler-Moix AL, Dolci U, Berger-Gryllaki M, Pannatier A, Cotting J, Di Paolo ER. Albuterol delivery in an in vitro pediatric ventilator lung model: comparison of jet, ultrasonic, and mesh nebulizers. Pediatr Crit Care Med 2013;14(2):e98-e102.

9. Dugernier J, Wittebole X, Roeseler J, Michotte JB, Sottiaux T, Dugernier $\mathrm{T}$, et al. Influence of inspiratory flow pattern and nebulizer position on aerosol delivery with a vibrating-mesh nebulizer during invasive mechanical ventilation: an in vitro analysis. J Aerosol Med Pulm Drug Deliv 2014 [Epub ahead of print] doi: 10.1089/ jamp.2014.1131.

10. O'Riordan TG, Greco MJ, Perry RJ, Smaldone GC. Nebulizer function during mechanical ventilation. Am Rev Respir Dis 1992;145(5): $1117-1122$.

11. Fink JB, Dhand R, Duarte AG, Jenne JW, Tobin MJ. Aerosol delivery from a metered-dose inhaler during mechanical ventilation. An in vitro model. Am J Respir Crit Care Med 1996;154(2 Pt 1): 382-387.

12. Chavez A, McCracken A, Berlinski A. Effect of face mask dead volume, respiratory rate, and tidal volume on inhaled albuterol delivery. Pediatr Pulmonol 2010;45(3):224-229.

13. Berlinski A. Effect of mask dead space and occlusion of mask holes on delivery of nebulized albuterol. Respir Care 2014;59(8):12281232.

14. Mouloudi E, Katsanoulas K, Anastasaki M, Hoing S, Georgopoulos D. Bronchodilator delivery by metered-dose inhaler in mechanically ventilated COPD patients: influence of tidal volume. Intensive Care Med 1999;25(11):1215-1221.

15. Hanhan U, Kissoon N, Payne M, Taylor C, Murphy S, DeNicola LK. Effects of in-line nebulization on preset ventilator variables. Respir Care 1993;38(5):474-478. 


\section{Aerosol Delivery During Pediatric Mechanical Ventilation}

16. O'Callaghan C, Clark AR, Milner AD. Why nebulise for more than five minutes? Arch Dis Child 1989;64(9):1270-1273.

17. Wan GH, Lin HL, Fink JB, Chen YH, Wang WJ, Chiu YC, et al. In vitro evaluation of aerosol delivery by different nebulization modes in pediatric and adult mechanical ventilators. Respir Care 2014; 59(10): 1494-1500.

18. Fink JB, Dhand R, Grychowski J, Fahey PJ, Tobin MJ. Reconciling in vitro and in vivo measurements of aerosol delivery from a me- tered-dose inhaler during mechanical ventilation and defining efficiency-enhancing factors. Am J Respir Crit Care Med 1999;159(1): 63-68.

19. Miller DD, Amin MM, Palmer LB, Shah AR, Smaldone GC. Aerosol delivery and modern mechanical ventilation: in vitro/in vivo evaluation. Am J Respir Crit Care Med 2003;168(10):1205-1209.

20. Alvine GF, Rodgers P, Fitzsimmons KM, Ahrens RC. Disposable jet nebulizers. How reliable are they? Chest 1992;101(2):316-319. 